\title{
Emerging urinary biomarkers in the diagnosis of acute kidney
}

\section{injury}

\author{
Prasad Devarajan \\ University of Cincinnati College of Medicine, Cincinnati Children's Hospital Medical Center, \\ Nephrology and Hypertension, MLC 7022, 3333 Burnet Avenue, Cincinnati, OH 45229, USA
}

\section{Abstract}

Background-Acute kidney injury (AKI) represents a common and devastating problem in clinical medicine. The lack of early biomarkers for AKI has led to a delay in initiating potentially effective therapies.

Objective-Identification of novel urinary biomarkers for AKI that have progressed to the clinical phase of the biomarker discovery process.

Methods-A literature review (PubMed, MedLine) from 2000 to the present.

Results/conclusions;-The most promising AKI biomarkers include neutrophil gelatinaseassociated lipocalin, IL-18, kidney injury molecule-1 and liver-type fatty acid binding protein. Studies to validate the sensitivity and specificity of these biomarkers in clinical samples from large cohorts and from multiple clinical situations are in progress, facilitated by the development of commercial tools for their measurement.

\section{Keywords}

acute kidney injury; acute renal failure; biomarker; IL-18; kidney injury molecule-1; liver-type fatty acid binding protein; nephrotoxicity; neutrophil gektinase-associated lipocalin

\section{The urgent need for acute kidney injury biomarkers}

Acute kidney injury (AKI) represents a complex disorder that occurs in a wide variety of clinical settings, with manifestations ranging from a minimal elevation in serum creatinine to anuric renal failure. AKI is a term proposed recently to reflect the entire spectrum of acute renal failure (ARF), and is characterized by 'an abrupt (within $48 \mathrm{~h}$ ) reduction in kidney function defined as an absolute increase in serum creatinine by either $>0.3 \mathrm{mg} / \mathrm{dl}$ or an increase of $>50 \%$ from baseline or oliguria of $<0.5 \mathrm{ml} / \mathrm{kg} / \mathrm{h}$ for $>6 \mathrm{~h}$ ' [1,2]. AKI represents a common but under-recognized problem in clinical medicine, with devastating immediate and long-term consequences [3-6]. The incidence of AKI varies from 5\% of hospitalized patients to $>30 \%$ of patients in intensive care units. There is substantial evidence that this incidence is increasing at an alarming rate, and the associated mortality and morbidity have remained unacceptably high despite technical improvements in clinical care [7-9]. Once established, there is no effective treatment for AKI, and the clinician can provide only supportive care with dialysis. Although the worst outcomes in AKI are encountered in patients requiring dialysis [10,11], even mild degrees of AKI as evidenced by small increases in serum creatinine foretell an increase in mortality and morbidity rate [12-15]. Although recent advances have suggested novel therapeutic approaches in animal models, translational efforts in humans have yielded 
disappointing results. A principal reason for this is the paucity of early biomarkers for AKI, leading to a delay in initiating potentially effective therapies [16-19].

In current clinical practice, AKI is typically diagnosed by a rise in serum creatinine. Unfortunately, creatinine is an unreliable indicator during acute changes in kidney function [20-23]. First, serum creatinine levels can vary widely with age, gender, muscle mass, muscle metabolism, medication and hydration status. Second, serum creatinine concentrations may not change until a substantial portion of kidneyfunction has already been lost, owing to a large renal reserve. Third, at lower rates of glomerular filtration, the enhanced tubular secretion of creatinine results in overestimation of renal function. Fourth, during acute changes in glomerular filtration, serum creatinine does not accurately depict kidney function until steadystate equilibrium has been reached, which may occur only 2-3 days after the initiating injury. However, animal studies have shown that although AKI can be prevented and/or treated by several maneuvers, these must be instituted within a narrow window of opportunity, well before the serum creatinine even begins to rise [16-19]. Not surprisingly, the use of serum creatinine as a therapeutic trigger has resulted in the failure of landmark clinical trials investigating promising interventions for AKI in humans [24,25].

In addition to aiding in early diagnosis and prediction, biomarkers may serve several other purposes in AKI [26-29]. Thus, biomarkers are also needed for: i) identifying the primary location of injury (proximal tubule, distal tubule, interstitium, or vasculature); ii) pinpointing the duration of kidney failure (AKI, chronic kidney disease, or 'acute-on-chronic'); iii) discerning AKI subtypes (prerenal, intrinsic renal, or postrenal); iv) identifying AKI etiologies (ischemia, toxins, sepsis, or a combination); v) differentiating AKI from other forms of acute kidney disease (urinary tract infection, glomerulonephritis, interstitial nephritis); vi) risk stratification and prognostication (duration and severity of AKI, need for renal replacement therapy, length of hospital stay, mortality); vii) defining the course of AKI; and viii) monitoring the response to AKI interventions.

Desirable characteristics of clinically applicable AKI biomarkers include; i) they should be non-invasive and easy to perform at the bedside or in a standard clinical laboratory, using easily accessible samples such as blood or urine; ii) they should be rapidly and reliably measurable using a standardized assay platform; iii) they should be highly sensitive to facilitate early detection, and with a wide dynamic range and cutoff values that allow for risk stratification; iv) they should be highly specific for AKI, and enable the identification of AKI subtypes and etiologies; and v) they should exhibit strong biomarker properties on receiver-operating characteristic (ROC) curves.

\section{The search for novel acute kidney injury biomarkers}

The biomarker development process has typically been divided into five phases [30]. The first phase is the preclinical discovery phase, which requires high-quality, well-characterized tissue or body fluid samples from carefully chosen animal or human models of the disease under investigation. Typically, tissue analysis uses genomic approaches, whereas body fluids are best analyzed by proteomic techniques. Identifying biomarkers in the serum or urine is most desirable, as these samples are easily obtained and allow for non-invasive testing. Urine is more likely to contain biomarkers arising from the kidney, more applicable for easy patient self-testing, and more amenable to proteomic screening because of the limited number of protein species present. However, urine samples are more prone to protein degradation, and biomarker concentrations may be confounded by changes in urine flow rate. Serum samples are readily available, even in anuric patients, and serum biomarkers may exhibit better stability. On the other hand, serum markers may reflect the systemic response to a disease process rather than specific organ involvement, and the presence of a large number of normally abundant 
proteins (such as albumin and immunoglobulins) in blood renders proteomic approaches difficult. The widespread availability of enabling technologies such as functional genomics and proteomics has accelerated the rate of novel biomarker discovery. Transcriptome profiling studies have identified several genes whose protein products have emerged as AKI biomarkers [31]. However, microarray-based methods cannot be used for the direct analysis of biological fluids, and usually require downstream confirmation by proteomic techniques before clinical use. Proteomics is the study of both the structure and function of proteins by a variety of methods, such as gel electrophoresis, immunoblotting, mass spectrometry, and enzymatic or metabolic assays. Advancing technologies have radically improved the speed and precision of identifying and measuring proteins in biological fluids, and proteomic approaches are also beginning to yield new AKI biomarkers [32-34].

Phase II is the assay development phase, during which a reliable and reproducible assay is developed, optimized and tested on existing samples of an established disease state. In the case of AKI, this has typically involved generating antibodies to identified biomarkers and testing biomarker concentrations in the urine or plasma of patients with established AKI using standardized platforms such as ELISA. Phase III is the retrospective study stage, in which the biomarker is tested in samples from completed clinical trials, to differentiate populations of interest and to test whether the biomarker can detect the disease before existing gold standards (in this case, before an increase in serum creatinine). Phase IV is the prospective screening stage, during which the biomarker is used to screen a population to identify extent and characteristics of the disease and the critical biomarker properties (including sensitivity/ specificity and area under the ROC curve). Phase V is the final stage, which determines the impact of screening on reducing disease burden and improving the outcome.

The quest to improve the early diagnosis of AKI is an area of intense contemporary research [26-29]. Conventional urinary biomarkers such as casts and fractional excretion of sodium have been insensitive and nonspecific for the early recognition of AKI. Other traditional urinary biomarkers such as filtered high molecular mass proteins and tubular proteins or enzymes have also suffered from lack of specificity and a dearth of standardized assays. Fortunately, the application of innovative technologies such as functional genomics and proteomics to human and animal models of kidney disease has uncovered several new candidates that are emerging as biomarkers of AKI. Several of these candidates have now progressed through the first three stages of the biomarker development process, and have entered Phase IV This review updates the reader on the current status of new urinary biomarkers of AKI that hold the greatest promise and potential to reach Phases IV and V in the biomarker development process. At present, the main contenders include neutrophil gelatinase-associated lipocalin (NGAL), IL-18, kidney injury molecule-1 (KIM-1) and liver-type fatty acid binding protein (L-FABP).

\section{Neutrophil gelatinase-associated lipocalin as an acute kidney injury biomarker}

The preclinical discovery phase of the AKI biomarker development process has been facilitated by transcriptome profiling technologies. Several investigators have used molecular techniques such as cDNA microarrays and subtractive hybridizations combined with downstream proteomic analysis to identify new biomarkers in AKI. The first detailed mouse kidney microarray analysis performed at early time points after AKI revealed the gene encoding neutrophil gelatinase-associated lipocalin ( $\mathrm{Ngal}$, also known as $l \mathrm{cn} 2)$ to be one of the most upregulated transcripts in the kidney [35], a finding that has now been confirmed in several other transcriptome profiling studies [36-39). Downstream proteomic studies have also revealed NGAL to be one of the earliest and most robustly induced proteins in the kidney after ischemic or nephrotoxic AKI in animal models, and NGAL protein is easily detected in the 
blood and urine soon after AKI [40-43]. These robust and reproducible findings have spawned several translational proteomic studies to evaluate NGAL as a new biomarker in human AKI.

The initial assay development phase of NGAL as an AKI biomarker was pioneered by the development of a reproducible ELISA assay for human NGAL [44]. The assay was found to be sensitive down to the picomolar range, and usable for both urine and plasma with excellent inter- and intra-assay coefficient variations in the 5-10\% range, thus setting the stage for Phase III studies. An ELISA assay for NGAL is now commercially available from Antibody Shop (Grusbakken, Denmark).

In a cross-sectional study, subjects in the intensive care unit with established AKI displayed > 100 -fold increase in urine NGAL by western blotting when compared with normal controls [42]. Urine NGAL levels correlated highly with serum creatinine levels. Kidney biopsies in these patients showed intense accumulation of immunoreactive NGAL in 50\% of the cortical tubules. These results therefore identified NGAL as a widespread and sensitive response to established AKI in humans.

In a prospective study of 71 children undergoing cardiopulmonary bypass, AKI (defined as a $50 \%$ increase in serum creatinine) occurred in $28 \%$ of the subjects, but the diagnosis using serum creatinine was possible only 1-3 days after surgery [44]. In marked contrast, NGAL measurements by western blotting and by ELISA revealed a robust 10 -fold or more increase in the urine and plasma, within $2-6 \mathrm{~h}$ of the surgery in patients who subsequently developed AKI. Both urine and plasma NGAL were powerful independent predictors of AKI, with an area under the receiver-operating characteristic curve (AUC) of 0.998 for the $2 \mathrm{~h}$ urine NGAL and 0.91 for the $2 \mathrm{~h}$ plasma NGAL measurement [44], The $2 \mathrm{~h}$ urine NGAL level represented a strong independent predictor of clinical outcomes such as duration of AKI among cases [45]. In a subsequent limited prospective validation study, urine NGAL was measured at 4, 12 and $24 \mathrm{~h}$ after cardiac surgery in 40 children, out of which 21 subjects developed AKI 2-3 days after the operation [46]. The area under the NGAL ROC curve at $4 \mathrm{~h}$ post surgery was 1.000 . Derived sensitivities and specificities for a urine NGAL cutpoint concentration of $100 \mathrm{ng} / \mathrm{mg}$ creatinine yielded both perfect sensitivity (1.000) and perfect specificity (1.000) for the prediction of AKI [46]. Thus, NGAL emerged as a sensitive, specific and highly predictive early biomarker of AKI in the urine and plasma, after cardiac surgery in children. These findings have now been confirmed in a prospective study of adults who developed AKI after cardiac surgery, in whom urinary NGAL was significantly elevated $1-3 \mathrm{~h}$ after the operation [47]. AKI, defined as a 50\% increase in serum creatinine, did not occur until the third postoperative day. However, patients who did not encounter AKI also displayed a significant increase in urine NGAL in the early postoperative period, although to a much lesser degree than those who subsequently developed AKI. The AUC reported in this study was 0.74 for the $3 \mathrm{~h} \mathrm{NGAL}$ and 0.80 for the $18 \mathrm{~h} \mathrm{NGAL}$, which is perhaps reflective of the confounding variables typically encountered in adults.

NGAL has also been evaluated as a biomarker of AKI in kidney transplantation. Biopsies of kidneys obtained $1 \mathrm{~h}$ after vascular anastomosis revealed a significant correlation between NGAL staining intensity and the subsequent development of delayed graft function [48]. In a prospective multicenter study of children and adults, urine NGAL levels in samples collected on the day of transplant clearly identified cadaveric kidney recipients who subsequently developed delayed graft function and dialysis requirement (which typically occurred 2-4 days later). The ROC curve for prediction of delayed graft function based on urine NGAL at day 0 showed an AUC of 0.9, indicative of an excellent predictive biomarker [49]. In a recent retrospective study of kidney transplant patients undergoing either protocol biopsies or clinically indicated biopsies, urine NGAL measurements were found to be significantly increased in subjects with tubulitis or other tubular pathologies [50]. Urine NGAL also tended 
to be increased in subjects with subclinical tubulitis $(\mathrm{p}=0.06)$, raising the possibility of NGAL representing a non-invasive screening tool for the detection of tubulo-interstitial disease in the early months following kidney transplantation.

Several investigators have examined the role of NGAL as a predictive biomarker of nephrotoxicity following contrast administration, with promising results [51-54]. In a prospective study of children undergoing elective cardiac catheterization with contrast administration, both urine and plasma NGAL predicted contrast-induced nephropathy (defined as a $50 \%$ increase in serum creatinine from baseline) within $2 \mathrm{~h}$ of contrast administration [54]. Using a cutoff value of $100 \mathrm{ng} / \mathrm{ml}$, the AUC for prediction of contrast nephropathy was excellent for the $2 \mathrm{~h}$ urine NGAL (0.92) as well as the $2 \mathrm{~h}$ plasma NGAL (0.91). By multivariate analysis, the $2 \mathrm{~h}$ NGAL concentrations in the urine and plasma were found to be powerful independent predictors of contrast nephropathy [54]. In studies of adults administered contrast, early rises in both urine $(4 \mathrm{~h})$ and plasma $(2 \mathrm{~h})$ NGAL were documented, in comparison with a much later increase in plasma cystatin $\mathrm{C}$ levels (8-24 $\mathrm{h}$ after contrast administration), providing further support for NGAL as an early biomarker of contrast nephropathy [51-53].

Urine NGAL obtained early during the hospitalization has also been shown to predict the severity of AKI and dialysis requirement in a multi-center study of children with diarrheaassociated hemolytic uremic syndrome, with high sensitivity but low specificity [55]. Using a cutoff of $200 \mathrm{ng} / \mathrm{ml}$, NGAL in urine obtained soon after hospitalization was significantly increased in those children who subsequently developed severe AKI requiring dialysis. Recently published results also suggest that urine NGAL measurements represent early biomarkers of AKI in the intensive care setting, being able to predict this complication about 2 days before the rise in serum creatinine [56]. Early urine NGAL measurements were also predictive (albeit weakly) of duration of AKI as well as worsening of AKI in critically ill subjects [56]. Thus, NGAL is a useful early AKI marker that predicts development and severity of AKI even in a heterogeneous group of patients with unknown timing of kidney injury.

All results described thus far were obtained using research-based assays, which is not a practical option in the clinical setting. The availability of validated clinical tools for NGAL measurements could revolutionize renal diagnostics. In this regard, a standardized point-ofcare kit has been devised for the measurement of plasma NGAL (Triage ${ }^{\circledR}$ NGAL device, Biosite Incorporated). In a pilot study with 40 plasma samples and 12 calibration standards, NGAL measurements by research ELISA and by the Triage ${ }^{\circledR}$ NGAL device were highly correlated $(\mathrm{r}=0.94)$. In a subsequent study of 120 patients undergoing cardiopulmonary bypass surgery $(\mathrm{CPB})$, AKI ( $50 \%$ or greater increase in serum creatinine) developed in 45 patients, but the diagnosis using serum creatinine was delayed by $2-3$ days after CPB [57]. In contrast, mean plasma NGAL levels measured by the Triage ${ }^{\circledR}$ NGAL device increased threefold within $2 \mathrm{~h}$ of $\mathrm{CPB}$, and remained significantly elevated for the duration of the study. By multivariate analysis, plasma NGAL at $2 \mathrm{~h}$ postCPB time was the most powerful independent predictor of AKI ( $\mathrm{p}<0.0001$ ). For the $2 \mathrm{~h}$ plasma NGAL measurement, the area under the curve was 0.96 , sensitivity was 0.84 and the specificity was 0.94 for prediction of AKI using a cutoff value of $150 \mathrm{ng} / \mathrm{ml}$. The $2 \mathrm{~h}$ postoperative plasma NGAL levels correlated strongly with change in creatinine $(r=0.46, p<0.001)$, duration of AKI $(r=0.57, p<0.001)$ and length of hospital stay $(r=0.44, p<0.001)$, The $12 \mathrm{~h}$ plasma NGAL correlated strongly with mortality $(r=0.48$, $\mathrm{p}=0.004$ ) and all measures of morbidity mentioned above, Thus, accurate measurements of plasma NGAL are obtained using the point-of-care Triage ${ }^{\circledR}$ NGAL device. The assay is facile and performed on the Triage meter with quantitative results available within $15 \mathrm{~min}$, and requires only microliter quantities of whole blood or plasma. The assay is auto-calibrated and includes reactive internal controls that run with every sample applied. The assay is deployable directly to the point of patient care. Using this device, plasma NGAL was found to be an early predictive biomarker of AKI, morbidity and mortality after CPB [57]. 
In addition, a urine NGAL immunoassay has been developed for a standardized clinical platform (ARCHITECT ${ }^{\circledR}$ analyzer, Abbott Diagnostics). The development of the Abbott ARCHITECT $^{\circledR}$ urine NGAL assay has progressed through research assay prototype testing and has successfully met all research assay performance requirements developed by an expert panel of nephrologists and laboratory personnel. As a result of the assay performance observed in these studies, the assay design has transitioned into Abbott's formal product development process. In a pilot study with 136 urine samples and 6 calibration standards, NGAL measurements obtained by research ELISA and by the ARCHITECT assay were highly correlated $(\mathrm{r}=0.99)$. In a subsequent study, 196 children undergoing CPB were prospectively enrolled, and serial urine NGAL measurements obtained by ARCHITECT assay [58]. AKI developed in 99 patients but the diagnosis using serum creatinine was delayed by 2-3 days after CPB. By contrast, mean urine NGAL levels increased 15-fold within $2 \mathrm{~h}$, and 25-fold at 4 and $6 \mathrm{~h}$ after CPB. For the $2 \mathrm{~h}$ urine NGAL measurement, the area under the curve was 0.95 , sensitivity was 0.82 and the specificity was 0.90 for prediction of AKI using a cutoff value of $100 \mathrm{mg} / \mathrm{ml}$. The $2 \mathrm{~h}$ urinary NGAL levels were found to be highest in subjects who had either an earlier rise in serum creatinine $(3689 \pm 294 \mathrm{ng} / \mathrm{mg}$ creatinine $)$ or needed renal replacement therapy $(5081 \pm 320 \mathrm{ng} / \mathrm{mg}$ creatinine $)$. The $2 \mathrm{~h}$ urine NGAL levels correlated highly with severity of AKI $(r=0.66, p<0.0001)$, duration of AKI $(r=0.73, p<0.0001)$, length of hospital stay $(\mathrm{r}=0.42, \mathrm{p}<0.0001)$, dialysis requirement $(\mathrm{r}=0.38, \mathrm{p}=0.01)$ and death $(\mathrm{r}=0.53, \mathrm{p}=$ 0.01). Thus, accurate measurements of urine NGAL are obtained using the ARCHITECT platform. This assay is easy to perform with no manual pretreatment steps, a first result available within $35 \mathrm{~min}$, and it requires only $150 \mu \mathrm{l}$ of urine. The $2 \mathrm{~h}$ urine NGAL measured by ARCHITECT assay was found to be an early predictive biomarker of AKI severity and mortality after CPB [58].

In summary, NGAL is emerging as a center-stage player in the AKI field, as a new predictive biomarker, for prominent inclusion in the urinary 'AKI Biomarker Panel'. It is acknowledged, however, that the studies published thus far have been small, in which NGAL appears to be most sensitive and specific in relatively uncomplicated patient populations with AKI. Plasma NGAL measurements may be influenced by several coexisting variables such as systemic infections [59], inflammatory conditions [60] and malignancies [61]; however, any relationship between plasma and urinary NGAL requires further clarification. Any NGAL systemically released from infection, inflammation, or malignantly transformed epithelia would be freely filtered by the kidney glomerulus but would be largely reabsorbed by efficient endocytic mechanisms in the proximal tubules $[62,63]$. Indeed, systemic injection of labeled exogenous recombinant NGAL, even in microgram quantities, has been shown to be rapidly and almost completely reabsorbed by the kidney, with minimal amounts if any appearing in the urine $[42,64]$. Thus, any urinary excretion of NGAL is likely to be present only when there is concomitant renal tubular injury that increases de novo NGAL synthesis and/or precludes NGAL reabsorption, In strong support of this notion is the finding from gene expression studies in AKI that rapid and massive upregulation of NGAL mRNA occurs in the thick ascending limb of Henle's loop and the collecting ducts, with resultant synthesis of NGAL protein in the distal nephron (the renal pool) and secretion into the urine where it comprises the major fraction of urinary NGAL $[62,63]$. In addition, AKI results in increased NGAL mRNA expression in distant organs, especially in the liver and lung, and the overexpressed NGAL protein is released into the circulation and constitutes the distinct systemic pool $[62,63]$.

There is an emerging literature suggesting that NGAL is also a marker of chronic kidney disease (CKD) and severity [65]. In one study of 33 subjects with CKD (from glomerulonephritides) and 20 controls, the mean urine NGAL levels were higher in CKD patients $(378.28 \pm 111.13$ versus $7.38 \pm 3.26 \mathrm{ng} / \mathrm{ml} ; \mathrm{p}=0.01)$. Furthermore, NGAL levels were significantly correlated with serum creatinine $(\mathrm{r}=0.588$, $\mathrm{p}$-value $=0.02)$, glomerular filtration rate $(\mathrm{GFR})(\mathrm{r}=-0.528$, p-value $=0.04)$ and proteinuria $(r=0.294$, $\mathrm{p}$-value $=0.01)[66]$. Both urine and plasma NGAL 
represent biomarkers of CKD severity in patients with autosomal dominant polycystic kidney disease [67]. In these subjects, urine and plasma NGAL measurements correlated with residual GFR, and those with greater severity of cystic disease (measured as number of cysts $>10$ ) displayed the highest NGAL values [67]. Urine NGAL has also been shown to represent an early biomarker for the degree of chronic injury in patients with IgA nephropathy [68] and lupus nephritis [69]. These observations are important to bear in mind when using NGAL as a biomarker of AKI, since the risk of AKI is increased in patients with underlying CKD.

\section{IL-18 as an acute kidney injury biomarker}

IL-18 is a pro-inflammatory cytokine that is known to be induced and cleaved in the proximal tubule, and subsequently easily detected in the urine following ischemic AKI in animal models [70]. In a cross-sectional study, urine IL-18 levels measured by a sensitive commercially available ELISA (Medical and Biological laboratories, Nagoya, Japan) were markedly elevated in patients with established AKI but not in subjects with urinary tract infection, chronic kidney disease, nephrotic syndrome, or prerenal azotemia [71], In a subsequent study, urinary IL-18 was found to be significantly upregulated before the increase in serum creatinine in patients with acute respiratory distress syndrome who develop AKI [72]. On multivariate analysis, urine IL-18 levels $>100 \mathrm{pg} / \mathrm{mg}$ creatinine predicted the development of AKI $24 \mathrm{~h}$ before the rise in serum creatinine, with an adjusted odds ratio of 6.5 and an AUC of 0.73 . Urine IL-18 on the day of initiation of mechanical ventilation was also predictive of mortality in these patients, independent of severity of illness scores and serum creatinine [72].

Both urinary IL-18 and NGAL were recently shown to represent early, predictive, sequential AKI biomarkers in children undergoing cardiac surgery [45]. In patients who developed AKI $2-3$ days after surgery, urinary NGAL was induced within $2 \mathrm{~h}$ and peaked at $6 \mathrm{~h}$, whereas urine IL-18 levels increased around $6 \mathrm{~h}$ and peaked at $>25$-fold at $12 \mathrm{~h}$ post surgery (AUC 0.75). Both IL-18 and NGAL were independently associated with duration of AKI among cases.

In subjects who undergo kidney transplantation, urine IL-18 and NGAL have emerged as predictive biomarkers for delayed graft function (defined as dialysis requirement within the first week after transplantation) [49]. In a prospective multi-center study of children and adults, both IL-18 and NGAL in urine samples collected on the day of transplant predicted subsequent delayed graft function and dialysis requirement with an AUC of 0.9 for both biomarkers. By multivariate analysis, both urine IL-18 and NGAL predicted the trend in serum creatinine in the post-transplant period after adjusting for age, gender, race, urine output and cold ischemia time $(\mathrm{p}<0.01)$.

Recently published results also suggest that urine IL-18 measurements represent early biomarkers of AKI in the intensive care setting, being able to predict this complication about 2 days before the rise in serum creatinine [73]. Early urine IL-18 measurements correlated with the severity of AKI as well as mortality. Thus, urinary IL-18 rises before serum creatinine in non-septic critically ill children, predicts severity of AKI and is an independent predictor of mortality in this heterogeneous group of patients with unknown timing of kidney injury [73].

Thus, IL-18 may also represent a promising candidate for inclusion in the urinary 'AKI Biomarker Panel'. IL-18 is more specific to ischemic AKI, and largely not affected by nephrotoxins, chronic kidney disease or urinary tract infections. It is likely that NGAL and IL-18 will emerge as sequential urinary biomarkers of AKI. However, IL-18 measurements may also be influenced by a number of coexisting variables, since renal IL-18 mRNA levels are known to be induced in other disease states such as endotoxemia, immunologic injury and cisplatin toxicity [74]. Furthermore, plasma IL-18 levels are known to be increased in various pathophysiologic states, such as inflammatory arthritis, inflammatory bowel disease, systemic 
lupus erythetnatosus, psoriasis, hepatitis and multiple sclerosis. The relationship between plasma and urine IL-18 remains largely unexplored.

\section{Kidney injury molecule-1 as an acute kidney injury biomarker}

Kidney injury molecule 1 (Kim- 1 ) was first identified by subtractive hybridization screening as a gene that is markedly upregulated in ischemic rat kidneys [75], a finding that has been confirmed in several other transcriptome profiling studies. Downstream proteomic studies have also shown KIM-1 to be one of the most highly induced proteins in the kidney after AKI in animal models, and a proteolytically processed domain of KIM-1 is easily detected in the urine soon after AKI [76-78]. Assays for KIM-1 (ELISA and microbead-based) have been developed in research laboratories [79] but are not commercially available.

In a small human cross-sectional study, KIM-1 was found to be markedly induced in proximal tubules in kidney biopsies from patients with established AKI (primarily ischemic), and urinary KIM-1 measured by ELISA distinguished ischemic AKI from prerenal azotemia and chronic renal disease [76]. Recent studies have expanded the potential clinical use of KIM-1 as a predictive AKI biomarker. In a case-control study of 40 children undergoing cardiac surgery, 20 with AKI (defined as a 50\% increase in serum creatinine) and 20 without AKI, urinary KIM-1 levels were markedly enhanced, with an AUC of 0.83 at the $12 \mathrm{~h}$ time point for predicting AKI [80]. In a larger prospective cohort study of 201 hospitalized patients with established AKI, both urinary KIM- 1 as well as urinary $N$-acetyl- $\beta$-(D)-glucosaminidase (NAG) were found to be associated with adverse clinical outcomes, including dialysis requirement and death [81]. However, the association between KIM-1 concentrations and the composite end point (dialysis or mortality) was weak (odds ratio 3.2 for highest versus lowest quartile), and was not significant after adjustment for covariates.

Thus, KIM-1 represents a promising candidate for inclusion in the urinary 'AKI Biomarker Panel'. It is likely that NGAL and KIM-1 will emerge as tandem biomarkers of AKI, with NGAL being most sensitive at the earliest time points and KIM-1 potentially adding specificity at slightly later time points. One advantage of KIM-1 as a urinary biomarker is the fact that its expression seems to be limited to the injured or diseased kidney, and no systemic source of KIM-1 has been described; however, urinary KIM-1 measurements may be influenced by some other confounding variables. KIM-1 is induced in the kidney and upregulated in the urine by a large number of nephrotoxins, including ciclosporin, cisplatin, cadmium, gentamicin, mercury and chromium [82]. Similarly, KIM-1 in the kidney and urine is induced in a variety of chronic proteinuric, inflammatory and fibrotic disease states in humans [83].

\section{Liver-type fatty acid binding protein an as acute kidney injury biomarker}

Liver fatty acid binding protein is a 14-kDa protein normally expressed in the kidney proximal convoluted and straight tubules [84]. In a model of cisplatin-induced AKI, there was increased shedding of urinary L-FABP within the first $24 \mathrm{~h}$, whereas a rise in serum creatinine was not detectable until after $72 \mathrm{~h}$ of cisplatin treatment [85]. A two-step sandwich ELISA method using monoclonal antibodies to human L-FABP protein was established for quantification of L-FABP in urine [86,87], and is now commercially available (CMIC Co. Ltd, Tokyo, Japan). One pilot study examined the role of urinary L-FABP in contrast-induced AKI, defined as an increase in serum creatinine of $>0.5 \mathrm{mg} / \mathrm{dl}$ or a relative increase of $>25 \%$ at $2-5$ days after the procedure [88]. Urinary L-FABP levels were significantly increased before the increase in serum creatinine only in those patients that developed AKI post contrast dye $(n=13)$. In a recent study involving 12 living-related kidney transplant patients immediately after reperfusion of their transplanted organs [89], a significant direct correlation was found between urinary L-FABP level and both peritubular capillary blood flow and the ischemic time of the transplanted kidney (both $\mathrm{p}<0.0001$ ), as well as hospital stay $(\mathrm{p}<0.05)$. 
In a recent prospective study of 40 children undergoing cardiac surgery, 21 developed AKI (defined as a 50\% increase in serum creatinine from baseline, which typically occurred 2-3 days post surgery). In this group, ELISA analysis showed increased urine L-FABP from levels before surgery of $20 \pm 4$ to $1885 \pm 500 \mathrm{ng} / \mathrm{mg}$ creatinine at $4 \mathrm{~h}$ [90]. Western blot analysis confirmed the presence of urinary L-FABP. Urinary L-FABP levels at $4 \mathrm{~h}$ post cardiac surgery was a powerful independent risk indicator for AKI. For concentration of urinary L-FABP at 4 $\mathrm{h}$, the AUC was 0.810 for a cutoff value of $486 \mathrm{ng} / \mathrm{mg}$ creatinine.

Thus, L-FABP also appears to be a promising candidate for inclusion in the urinary 'AKI Biomarker Panel'. It is possible that NGAL and L-FABP will emerge as the earliest biomarkers of AKI, with KIM-1 and IL-18 potentially adding specificity at slightly later time points. However, urinary L-FABP measurements may also be influenced by several confounding variables [91]. Several studies have documented increased urinary L-FABP levels in patients with non-diabetic chronic kidney disease [92], early diabetic nephropathy [93], idiopathic focal glomerulosclerosis [94] and polycystic kidney disease [95]. Another issue worth considering is the fact that L-FABP is also abundantly expressed in the liver, and urinary L-FABP may be influenced by serum L-FABP levels. As in the case of NGAL, any systemic L-FABP would be freely filtered by the kidney glomerulus but would largely be reabsorbed by megalinmediated proximal tubular uptake [96], Encouragingly, studies have shown that in the presence of chronic renal disease, a reduction in proximal tubular reabsorption results in increased urinary L-FABP excretion, which does not quantitatively correlate with serum L-FABP levels [97]. The estimated contribution of serum L-FABP to urinary L-FABP in chronic kidney disease was only $~ 3 \%$, suggesting that serum L-FABP levels do not influence urinary L-FABP levels. Further evidence for this notion has recently been provided in patients who developed AKI post cardiac surgery, who also developed significant early acute liver injury [90], In this subgroup, there was a significant increase in serum L-FABP levels at $12 \mathrm{~h}$ post cardiac surgery, but not at $4 \mathrm{~h}$. By contrast, urinary L-FABP levels measured in this same subset of patients were dramatically increased within the first $4 \mathrm{~h}$ post surgery, while urinary levels at $12 \mathrm{~h}$ had actually begun to decline. These findings confirm the dissociation between plasma and urine L-FABP levels in AKI, and suggest that increased urinary L-FABP levels at $4 \mathrm{~h}$ post cardiac surgery in AKI patients represent an enhanced shedding of L-FABP from the proximal tubule rather than reflecting increased filtration of high serum levels.

\section{Conclusion}

The tools of contemporary science are providing us with new biomarkers for the clinical investigation of AKI in humans. The most promising of these are chronicled in this review and summarized in Table 1. These include NGAL, IL-18, KIM-1 and L-FABP. As they represent tandem biomarkers, it is likely that the AKI panels will be useful for timing the initial insult and assessing the duration and severity of AKI. Based on the differential expression of the biomarkers, it is also likely that the AKI panels will help distinguish between the various types and etiologies of AKI, and predict clinical outcomes. However, they have hitherto been tested only in small studies and in a limited number of clinical situations. Studies to validate the sensitivity and specificity of these biomarkers in clinical samples from large cohorts and from multiple clinical situations are now in progress, facilitated by the development of commercial tools for the reproducible measurement of these biomarkers across different laboratories. Such clinical laboratory tools will be indispensable for the early diagnosis and initiation of timely therapeutic measures in AKI, a common clinical condition with a dismal prognosis.

\section{Expert opinion}

The incidence of AKI varies from 5\% of hospitalized patients to $>30 \%$ of patients in intensive care units, and it is rising. The associated mortality and morbidity have remained high. Once 
established, there is no effective treatment for human AKI. Ironically, even tragically, animal studies have shown that AKI can be prevented and/or treated by several maneuvers but these must be initiated within a narrow window of opportunity. Therein lies the Achilles' heel of AKI management - the paucity of early biomarkers has led to a delay in initiating potentially effective therapies in humans.

It is instructive to consider an analogous clinical situation, namely acute myocardial infarction (AMI), the medical evaluation of which has progressed over the past few decades from detection of Q-waves by electrocardiogram through a series of serum biomarkers with increasing sensitivity and predictive value. Now widely available to the clinician is a panel of tandem serum biomarkers that can not only establish the diagnosis with accuracy, but can also provide information on severity and duration of disease (Figure 1). This has allowed for timely institution of several therapeutic interventions, with a resultant $50 \%$ or so reduction in mortality rate. In stark comparison, the diagnosis, treatment and and prognosis of AKI have not changed appreciably in the last five decades. Utilizing serum creatinine measurements to institute promising interventions for AKI in humans is futile, and is analogous to waiting for $2-3$ days before intervening in patients with ischemic acute myocardial infarction or acute neurologic stroke. Identification of new AKI biomarkers has been designated as a top priority by the American Society of Nephrology [98]. The concept of developing a new toolbox for earlier diagnosis of disease states is also featured prominently in the NIH Roadmap for biomedical research [99].

Fortunately, we are closing in on a proposed 'AKI Urinary Biomarker Panel'. Incredibly, much of the confusion surrounding the early diagnosis of AKI is being solved by the adaptive response of the stressed kidney itself, with the rapid and robust induction of select genes whose protein products have provided us with highly promising biomarkers [100]. This includes NGAL, IL-18, KIM-1 and L-FABP, as illustrated in Figure 1. The availability of commercial ELISA-based tools for the measurement of most of these candidate biomarkers has allowed for widespread confirmation of the utility of these biomarkers in several AKI situations. All of these may now be considered to have completed the Phase III stage, and entered Phase IV (prospective screening stage) in the biomarker development process. Each individual biomarker in the putative AKI panel has merits and demerits, as detailed.

Within this panel, NGAL represents the most extensively validated and promising biomarker for AKI. As a result, robust and standardized clinical platforms for the accurate measurement of human NGAL have been developed by industry. The NGAL assays have successfully met all performance requirements and have transitioned into the formal product development process. It is hoped that these tools will provide clinicians with new methods for the early diagnosis of AKI, for the early initiation of potentially effective therapies, and for the monitoring of the efficacy of therapy.

It is doubtful, however, that any single biomarker such as NGAL will suffice in AKI. The reason for this is evident from the complex and heterogeneous nature of AKI, occurring in a wide variety of clinical settings with multiple pathophysiologic mechanisms that interplay with and amplify each other. It will probably require a panel of validated biomarkers to provide us with all the desired information. In addition to early diagnosis and prediction, biomarkers may serve to discern AKI subtypes. For example, the combination of NGAL and IL-18 may be useful for the diagnosis of contrast-induced nephropathy, because NGAL (but not IL-18) is induced early in this condition. Furthermore, urinary biomarker panels could identify etiologies, predict clinical outcomes (duration and severity of AKI, need for renal replacement therapy, length of hospital stay, mortality), allow for risk stratification and prognostication, and monitor the response to interventions, 
The availability of such information has the potential to revolutionize renal and critical care, and allow for the practice of personalized and predictive medicine at an unprecedented level. At the very least, clinicians informed of changes in urinary biomarkers would be aware of the potential for development of clinical AKI, and biomarkers could add substantively to existing clinical scoring systems for AKI prediction. Such patients would deserve closer monitoring with respect to blood pressure, urine output and renal perfusion. Every effort to monitor intravascular status and to optimize hydration and renal perfusion would be deployed. These subjects would benefit from the diligent avoidance of nephrotoxins. The ability to predict which patients will develop AKI could enable early initiation of potential interventions. For example, earlier intervention with renal replacement therapy may be strongly considered for subjects with elevated biomarker levels who are developing fluid overload but will not display increased serum creatinine for several days due to hemodilution and time required for re-establishment of a steady-state. The availability of promising early biomarkers may allow the timely initiation of interventions such as atrial natriuretic peptide and insulin-like growth factor that have been successful in smaller, Phase II-level efficacy studies but not in larger Phase III trials. Possibly, these interventions would have been successful if they had been initiated at the onset of AKI (as determined by predictive biomarkers \}, rather than waiting several days for serum creatinine to rise. In addition, animal studies have identified, and continue to reveal, new therapies such as growth factors, antiapoptotic, anti-inflammatory and antioxidant approaches that are effective in early AKI, before the rise in serum creatinine. The availability of standardized commercial platforms for urinary biomarker determination may allow these promising agents to be investigated in humans with AKI.

\section{Acknowledgements}

Declaration of interest

Studies cited in this review that were performed by the author's laboratory were supported by grants from the NIH (R01 DK53289 and R21 DK070163). Biosite ${ }^{\circledR}$ Incorporated has signed an exclusive licensing agreement with Cincinnati Children's Hospital for developing plasma NGAL as a biomarker of acute renal failure. Abbott Diagnostics has signed an exclusive licensing agreement with Cincinnati Children's Hospital for developing urine NGAL as a biomarker of acute renal failure.

\section{Bibliography}

Papers of special note have been highlighted as either of interest $(\bullet)$ or of considerable interest $(\bullet \bullet)$ to readers.

1. Bagga A, Bakkaloglu A, Devarajan P, et al. Improving outcomes from acute kidney injury: report of an initiative. Pediatr Nephrol 2007;22:1655-8165. [PubMed: 17665221]

2. Mehta RL, Kellum JA, Shah SV, et al. Acute Kidney Injury Network: report of an initiative to improve outcomes in acute kidney injury. Crit Care 2007;11:R31. [PubMed: 17331245]

3. Lameire N, Van Biesen W, Vanholder R. Acute renal failure. Lancet 2005;365:417-30. [PubMed: 15680458]

4. Uchino S, Kellum JA, Bellomo R, et al. Acute renal failure in critically ill patients: a multinational, multicenter study. JAMA 2005;294:813-8. [PubMed: 16106006]

5. Palevsky PM. Epidemiology of acute renal failure: the tip of the iceberg. Clin J Am Soc Nephrol 2006;1:6-7. [PubMed: 17699185]

6. Liangos O, Wald R, O'Bell JW, et al. Epidemiology and outcomes of acute renal failure in hospitalized patients: a national survey. Clin J Am Soc Nephrol 2006;1:43-51. [PubMed: 17699189]

7. Xue JL, Daniels F, Star RA, et al. Incidence and mortality of acute renal failure in medicare beneficiaries, 1992 to 2001. J Am Soc Nephrol 2006;17:1135-2. [PubMed: 16495381]

8. Ympa YP, Sakr Y, Reinhart K, et al. Has mortality from acute renal failure decreased? A systematic review of the literature. Am J Med 2005;118:827-32. [PubMed: 16084171] 
9. Waikar SS, Curhan GC, Wald R, et al. Declining mortality in patients with acute renal failure, 1988 to 2002. J Am Soc Nephrol 2006;17:H43-50.

10. Metnitz PG, Krenn CG, Steltzer H, et al. Effect of acute renal failure requiring renal replacement therapy on outcome in critically ill patients. Crit Care Med 2002;30:2051-8. [PubMed: 12352040]

11. Clermont G, Acker CG, Angus DC, et al. Renal failure in the ICU: comparison of the impact of acute renal failure and end-stage renal disease on ICU outcomes. Kidney Int 2002;62:986-96. [PubMed: 12164882]

12. Lassning A, Schmidlin D, Mouhieddine M, et al. Minimal changes of serum creatinine predict prognosis in patients after cardiothoracic surgery; a prospective cohort study. J Am Soc Nephrol 2004;15:1597-605. [PubMed: 15153571]

13. Levy MM, Macias WL, Vincent JL, et al. Early changes in organ function predict eventual survival in severe sepsis. Crit Care Med 2005;33:2194-201. [PubMed: 16215369]

14. Chettow GM, Burdick E, Honour M, et al. Acute kidney injury, mortality, length of stay, and costs in hospitalized patients. J Am Soc Nephrol 2005;16:3365-70. [PubMed: 16177006]

15. Hoste EAJ, Clermont G, Kersten A, et al. RIFLE criteria for acute kidney injury is associated with hospital mortality in critically ill patients: a cohort analysis. Crit Care 2006;10:R73-82. [PubMed: 16696865]

16. Schrier RW. Need to intervene in established acute renal failure. J Am Soc Nephrol 2004;15:27568. [PubMed: 15466282]

17. Hewitt SM, Dear J, Star RA. Discovery of protein biomarkers for renal diseases. J Am Soc Nephrol 2004;15:1677-89. [PubMed: 15213255]

18. Herget-Rosenthal S, Marggraf G, Hüsing J, et al. Early detection of acute renal failure by serum cystatin C. Kidney Int 2004;66:1115-22. [PubMed: 15327406]

19•. Devarajan P. Update on mechanisms of ischemic acute kidney injury. J Am Soc Nephrol 2006;17:1503-20. [PubMed: 16707563]A comprehensive update on the mechanisms underlying the pathogenesis of AKI.

20. Bellomo R, Kellum JA, Ronco C. Defining acute renal failure: physiological principles. Intensive Care Med 2004;30:33-7. [PubMed: 14618231]

21. Dennen P, Parikh CR. Biomarkers of acute kidney injury: can we replace serum creatinine? Clin Nephrol 2007;68:269-78. [PubMed: 18044258]

22. Ronco C. N-GAL: diagnosing AKI as soon as possible. Crit Care 2007;11(6):173. [PubMed: 18001501]

23. Goldstein SL. Kidney function assessment in the critically ill child: is it time to leave creatinine behind? Crit Care 2007;11(3):141. [PubMed: 17581273]

24. Allgren RL, Marbury TC, Rahman SN, et al. Anaritide in acute tubule necrosis. Auriculin Anaritide Acute Renal Failure Study Group. N Engl J Med 1997;336:828-34. [PubMed: 9062091]

25. Hirschberg R, Kopple J, Lipsett P, et al. Multicenter clinical trial of recombinant human insulin-like growth factor 1 in patients with acute renal failure. Kidney Int 1999;55:2423-32. [PubMed: 10354291]

26. Devarajan P. Emerging biomarkers of acute kidney injury. Contrib Nephrol 2007;156:203-12. [PubMed: 17464129]

27. Nguyen MT, Devarajan P. Biomarkers for the early detection of acute kidney injury. Pediatr Nephrol. 2007[Epub ahead of print]

28•. Devarajan P. Proteomics for biomarker discovery in acute kidney injury. Semin Nephrol 2007;27:637-51. [PubMed: 18061846]A comprehensive account of proteomic techniques that have resulted in new biomarker discovery in AKI

29. Parikh CR, Edelstein CL, Devarajan P, et al. Biomarkers of acute kidney injury: early diagnosis, pathogenesis, and recovery. J Investig Med 2007;55:333-40.

30 . Pepe MS, Etzioni R, Feng Z, et al. Phases of biomarker development for early detection of cancer. J Natl Cancer Inst 2001;93:1054-61. [PubMed: 11459866]A detailed account of the biomarker development process 
31. Devarajan P, Mishra J, Supavekin S, et al. Gene expression in early ischemic renal injury: clues towards pathogenesis, biomarker discovery, and novel therapeutics. Mol Genet Metab 2003;80:36576. [PubMed: 14654349]

32. Zhou H, Hewitt SM, Yuen PST, et al. Acute kidney injury biomarkers-needs, present status, and future promise. NephSAP 2006;5:63-71.

33. Perco P, Pleban C, Kainz A, et al. Protein biomarkers associated with acute renal failure and chronic kidney disease. Eur J Clin Invest 2006;36:753-63. [PubMed: 17032342]

34. Nguyen M, Ross G, Dent C, et al. Early prediction of acute renal injury using urinary proteomics. Am J Nephrol 2005;25:318-26. [PubMed: 15961952]

35. Supavekin S, Zhang W, Kucherlapati R, et al. Differential gene expression following early renal ischemia-reperfusion. Kidney Int 2003;63:1714-24. [PubMed: 12675847]

36. Keiran NE, Doran PP, Connolly SB, et al. Modification of the transcriptome response, to renal ischemia/reperfusion injury by lipoxin analog. Kidney Int 2003;64:480-92. [PubMed: 12846743]

37. Amin RP, Vickers AE, Sistare F, et al. Identification of putative gene based markers of renal toxicity. Environ Health Perspect 2004;112:465-79. [PubMed: 15033597]

38. Yuen PST, Jo S-K, Holly MK, et al. Ischemic and nephrotoxic acute renal failure are distinguished by their broad transcriptomic responses. Physiol Genomics 2006;25:375-86. [PubMed: 16507785]

39. Hung Y-C, Huang GS, Lin L-W, et al. Thea sinesis melanin prevents cisplatin-induced nephrotoxicity in mice. Food Chem Toxicol 2007;45:1123-30. [PubMed: 17303299]

40•. Mishra J, Ma Q, Prada A, et al. Identification of neutrophil gelatinase-associated lipocalin as a novel urinary biomarker for ischemic injury. J Am Soc Nephrol 2003;4:2534-43. [PubMed: 14514731] The first identification of NGAL as a biomarker of AKI in animal models

41. Mishra J, Mori K, Ma Q, et al. Neutrophil Gelatinase-Associated Lipocalin (NGAL): a novel urinary biomarker for cisplatin nephrotoxicity. Am J Nephrol 2004;24:307-15. [PubMed: 15148457]

42. Mori K, Lee HT, Rapoport D, et al. Endocytic delivery of lipocalin-siderophore-iron complex rescues the kidney from ischemia-reperfusion injury. J Clin Invest 2005;115:610-21. [PubMed: 15711640]

43. Devarajan R. Novel biomarkers for the early prediction of acute kidney injury. Cancer Ther 2005;3:477-88.

44••. Mishra J, Dent C, Tarabishi R, et al. Neutrophil gelatinase-associated lipocalin (NGAL) as a biomarker for acute renal injury following cardiac surgery. Lancet 2005;365:1231-8. [PubMed: 15811456]The first description of NGAL as a biomarker of AKI in humans

45. Parikh CR, Mishra J, Thiessen-Philbrook H, et al. Urinary IL-18 is an early predictive biomarker of acute kidney injury after cardiac surgery. Kidney Int 2006;70:199-203. [PubMed: 16710348]

46. Portilla D, Dent C, Sugaya T, et al. Liver Fatty Acid-Binding Protein as a biomarker of acute kidney injury after cardiac surgery. Kidney Int 2008;73(4):465-72. [PubMed: 18094680]

47. Wagener G, Jan M, Kim M, et al. Association between increases in urinary neutrophil gelatinaseassociated lipocalin and acute renal dysfunction after adult cardiac surgery. Anesthesiology 2006;105:485-91. [PubMed: 16931980]

48. Mishra J, Ma Q, Kelly C, et al. Kidney NGAL is a novel early marker of acute injury following transplantation. Pediatr Nephrol 2006;21:856-63. [PubMed: 16528543]

49•. Parikh CR, Jani A, Mishra J, et al. Urine NGAL and IL-18 are predictive biomarkers for delayed graft function following kidney transplantation. Am J Transplant 2006;6:1639-45. [PubMed: 16827865]The first description of NGAL and IL-18 as new predictive sequential biomarkers of AKI after kidney transplantation

50. Schaub S, Mayr M, Hönger G, et al. Detection of subclinical tubular injury after renal transplantation: comparison of urine protein analysis with allograft histopathology. Transplantation 2007;84:10412. [PubMed: 17627245]

51. Bachorzewska-Gajewska H, Malyszko J, Sitniewska E, et al. Neutrophil-gelatinase-associated lipocalin and renal function after percutaneous coronary interventions. Am J Nephrol 2006;26:28792. [PubMed: 16772710]

52. Bachorzewska-Gajewska H, Malyszko J, Sitniewska E, et al. Neutrophil gelatinase-associated lipocalin (NGAL) correlations with cystatin C, serum creatinine and eGFR in patients with normal serum creatinine undergoing coronary angiography. Nephrol Dial Transplant 2007;22:295-6. [PubMed: 16951419] 
53. Bachorzewska-Gajewska H, Malyszko J, Sitniewska E, et al. Could neutrophil-gelatinase-associated lipocalin and cystatin $\mathrm{C}$ predict the development of contrast-induced nephropathy after percutaneous coronary interventions in patients with stable angina and normal serum, creatinine values? Kidney Blood Press Res 2007;30:408-15. [PubMed: 17901710]

54•. Hirsch R, Dent C, Pfriem H, et al. NGAL is an early predictive biomarker of contrast-induced nephropathy in children. Pediatr Nephrol 2007;22:2089-95. [PubMed: 17874137]The first prospective study showing the use of NGAL as a predictive biomarker of AKI after contrast administration

55. Trachtman H, Christen E, Cnaan A, et al. Urinary neutrophil gelatinase-associated lipocalcin in D ${ }_{+}$HUS: a novel marker of renal injury. Pediatr Nephrol 2006;21:989-94. [PubMed: 16773412]

56•. Zappitelli M, Washburn KM, Arikan M, et al. Urine NGAL is an early marker of acute kidney injury in critically ill children. Grit Care 2007;11:R84. The first demonstration of NGAL as a predictive biomarker in a heterogeneous group of patients with unknown timing of kidney injury

57•. Dent CL, Ma Q, Dastrala S, et al. Plasma NGAL predicts acute kidney injury, morbidity and mortality after pediatric cardiac surgery: a prospective uncontrolled cohort study. Grit Care 2007;11:R127.The first validation of a point-of-care kit for NGAL measurement in humans

58. Bennett M, Dent CL, Ma Q, et al. Urine NGAL predicts severity of acute kidney injury after cardiac surgery: a prospective study. Clin J Am Soc Nephrol. 2008In press The first validation of a standardized clinical platform for NGAL measurement in humans

59. Xu SY, Pauksen K, Venge P. Serum measurements of human neutrophil lipocalin (HNL) discriminate between acute bacterial and viral infections. Scand J Clin Lab Invest 1995;55:125-31. [PubMed: 7667605]

60. Ohlsson S, Wieslander J, Segelmark M. Increased circulating levels of proteinase 3 in patients with antineutrophilic cytoplasmic autoantibodies-associated systemic vasculitis in remission. Clin Exp Immunol 2003;131:528-35. [PubMed: 12605707]

61. Devarajan P. Neutrophil gelatinase-associated lipocalin: new paths for an old shuttle. Cancer Ther 2007;5:463-70. [PubMed: 18449360]

62. Schmidt-Ott KM, Mori K, Kalandadze A, et al. Neutrophil gelatinase-associated lipocalin-mediated iron traffic in kidney epithelia. Curr Opin Nephrol Hypertens 2006;15:442-9. [PubMed: 16775460]

63. Schmidt-Ott KM, Mori K, Li JY, et al. Dual action of neutrophil gelatinase-associated lipocalin. J Am Soc Nephrol 2007;18:407-13. [PubMed: 17229907]

64. Mishra J, Mori K, Ma Q, et al. Amelioration of ischemic acute renal injury by neutrophil gelatinaseassociated lipocalin. J Am Soc Nephrol 2004;15:3073-82. [PubMed: 15579510]

65. Mitsnefes M, Kathman T, Mishra J, et al. Serum NGAL as a marker of renal function in children with chronic kidney disease. Pediatr Nephrol 2007;22:101-8. [PubMed: 17072653]

66. Bolignano D, Coppolino G, Campo S, et al. Urinary Neutrophil Gelatinase-Associated Lipocalin (NGAL) is associated with severity of renal disease in proteinuric patients. Nephrol Dial Transplant 2008;23(1):414-6. [PubMed: 17893105]

67. Bolignano D, Coppolino G, Campo S, et al. Neutrophil gelatinase-associated lipocalin in patients with autosomal-dominant polycystic kidney disease. Am J Nephrol 2007;27:373-8. [PubMed: 17570904]

68. Ding H, He Y, Li K, et al. Urinary neutrophil gelatinase-associated lipocalin (NGAL) is an early biomarker for renal tubulointerstitial injury in IgA nephropathy. Clin Immunol 2007;123:227-34. [PubMed: 17360238]

69. Brunner HI, Mueller M, Rutherford C, et al. Urinary NGAL as a biomarker of nephritis in childhoodonset SLE. Arthritis Rheum 2006;54:2577-84. [PubMed: 16868980]

70. Melnikov VY, Ecder T, Fantuzzi G, et al. Impaired IL-18 processing protects caspase-1 deficient mice from ischemic acute renal failure. J Clin Invest 2001;107:1145-52. [PubMed: 11342578]

71 . Parikh CR, Jani A, Melnikov VY, et al. Urinary interleukin-18 is a marker of human acute tubular necrosis. Am J Kidney Dis 2004;43:405-14. [PubMed: 14981598]This is the first study to demonstrate that IL-18 is a predictive biomarker in established AKL

72 . Parikh CR, Abraham E, Ancukiewicz M, et al. Urine IL-18 is an early diagnostic marker for acute kidney injury and predicts mortality in the intensive care unit. J Am Soc Nephrol 2005;16:304652. [PubMed: 16148039]This study shows the ability of IL-18 to predict adverse clinical outcomes 
73. Washburn KK, Zappitelli M, Arikan AA, et al. Urinary Interleukin-18 is an Acute Kidney Injury Biomarker in Critically III Children. Nephrol Dial Transplant 2008;23(2):566-72. [PubMed: 17911094]

74. Leslie JA, Meldrum KK. The role of interleukin-18 in renal injury. J Surg Res 2008;145(1):170-5. [PubMed: 17658553]

75•. Ichimura T, Bonventre JC, Bailly V, et al. Kidney Injury Molecule-1 (KIM-1), a putative epithelial cell adhesion molecule containing a novel immunoglobulin domain, is up-regulated in renal cells after injury. J Biol 1998;273:4135-42.This is the first paper to report KIM-1 as an AKI biomarker

76. Han WK, Bailly V, Abichandani R, et al. Kidney injury molecule-1 (KIM-1): a novel biomarker for human renal proximal tubule injury. Kidney Int 2006;62:237-44. [PubMed: 12081583]

77. Ichimura T, Hung CC, Yang SA, et al. Kidney injury molecule-1: a tissue and urinary biomarker for nephrotoxicant-induced renal injury. Am J Physiol Renal Physiol 2004;286:F552-63. [PubMed: 14600030]

78. Vaidya VS, Ramirez V, Ichimura T, et al. Urinary kidney injury molecule-1: a sensitive quantitative biomarker for early detection of kidney tubular injury. Am J Physiol Renal Physiol 2006;290:F51729. [PubMed: 16174863]

79. Vaidya VS, Ferguson MA, Bonventre JV. Biomarkers of acute kidney injury. Ann Rev Pharmacol Toxicol 2008;48:17.1-17.31.

80•. Han WK, Waikar SS, Johnson A, et al. Urinary biomarkers in the early diagnosis of acute kidney injury. Kidney Int. 2007[Epub ahead of print] This is the first study to report the value of KIM-1 for predicting AKI after cardiac surgery.

81 • Liangos O, Perianayagam MC, Vaidya VS, et al. Urinary N-Acetyl- $\beta$-(D)-Glucosaminidase activity and Kidney Injury Molecule-1 level are associated with adverse outcomes in acute renal failure. $\mathbf{J}$ Am Soc Nephrol 2007;18:904-12. [PubMed: 17267747]This is the first study to report the value of KIM-1 for predicting adverse clinical outcomes in patients with AKI

82. Zhou Y, Vaidya VS, Brown RP, et al. Comparison of kidney injury molecule-1 and other nephrotoxicity biomarkers in urine and kidney following acute exposure to gentamicin, mercury, and chromium. Toxicol Sci 2008;101:159-70. [PubMed: 17934191]

83. van Timmeren MM, van den Heuvel MC, Bailly V, et al. Tubular kidney injury molecule-1 (KIM-1) in human renal disease. J Pathol 2007;212:209-17. [PubMed: 17471468]

84. Maatman RGHJ, Van de Westerlo EMA, Van Kuppevelt THMSM, et al. Molecular identification of the liver- and the heart-type fatty acid-biding proteins in human and rat kidney. Biochem $\mathrm{J}$ 1992;288:285-90. [PubMed: 1280113]

85. Negishi K, Noiri E, Sugaya T, et al. A role of liver fatty acid binding protein in cisplatin-induced acute renal failure. Kidney Int 2007;72:348-58. [PubMed: 17495861]

86. Kamijo A, Kimura K, Sugaya T, et al. Urinary fatty acid-binding protein as a new clinical marker of the progression of chronic renal disease. J Lab Clin Med 2004;143:23-30. [PubMed: 14749682]

87. Kamijo A, Sugaya T, Hikawa A, et al. Urinary excretion of fatty acid-binding protein reflects stress overload on the proximal tubules. Am J Pathol 2004;165:1243-55. [PubMed: 15466390]

88. Nakamura T, Sugaya T, Node K, et al. Urinary excretion of liver-type fatty acid-binding protein in contrast medium-induced nephropathy. Am J Kidney Dis 2006;4:439-44. [PubMed: 16490622]

89•. Yamamoto T, Noiri E, Ono Y, et al. Renal L-type fatty acid-binding protein in acute ischemic injury. J Am Soc Nephrol 2007;18:2894-902. [PubMed: 17942962]This is the first report showing the value of urinary L-FABP in patients after kidney transplantation

90••. Portilla D, Dent C, Sugaya T, et al. Liver fatty acid-binding protein as a biomarker of acute kidney injury after cardiac surgery. Kidney Int. 2007[Epub ahead of print] This is the first report showing the value of urinary L-FABP for early prediction of AKI in patients after cardiac surgery

91. Kamijo-Ikemori A, Sugaya T, Kimura K. Urinary fatty acid binding protein in renal disease. Clin Chim Acta 2006;374:1-7. [PubMed: 16860300]

92. Kamijo A, Sugaya T, Hikawa A, et al. Clinical evaluation of urinary excretion of liver-type fatty acid binding protein as a marker for monitoring chronic kidney disease: a multi-center trial. J Lab Clin Med 2005;145:125-33. [PubMed: 15871303] 
93. Nakamura T, Sugaya T, Kawagoe Y, et al. Effect of pitavastatin on urinary Liver-type fatty acidbinding protein levels in patients with early diabetic nephropathy. Diabetes Care 2005;28:2728-32. [PubMed: 16249547]

94. Nakamura T, Sugaya T, Kawagoe Y, et al. Urinary liver-type fatty acid-binding protein levels for differential diagnosis of idiopathic focal glomerulosclerosis and minor glomerular abnormalities and effect of low-density lipoprotein apheresis. Clin Nephrol 2006;65:1-6. [PubMed: 16429835]

95. Nakamura T, Sugaya T, Kawagoe Y, et al. Candesattan reduces urinary liver-type fatty acid-binding protein excretion in patients with autosomal dominant polycystic kidney disease. Am J Med Sci 2005;330:161-5. [PubMed: 16234607]

96. Oyama Y, Takeda T, Hama H, et al. Evidence for megalin-mediated proximal tubular uptake of LFABP, a carrier of potentially nephrotoxic molecules. Lab Invest 2005;85:522-31. [PubMed: 15696188]

97. Kamijo A, Sugaya T, Hikawa A, et al. Urinary liver-type fatty acid binding protein as a useful biomarker in chronic kidney disease. Mol Cell Biochem 2006;284:175-82. [PubMed: 16532260]

98. American Society of Nephrology Renal Research Report. J Am Soc Nephrol 2005;16:1886-93. [PubMed: 15888557]

99. Zerhouni E. The NIH Roadmap. Science 2003;302:63-5. [PubMed: 14526066]

100. Devarajan P, Parikh CR, Barasch J. Case 31-2007: a man with abdominal pain and elevated creatinine. N Engl J Med 2008;358(3):312. [PubMed: 18203334] 


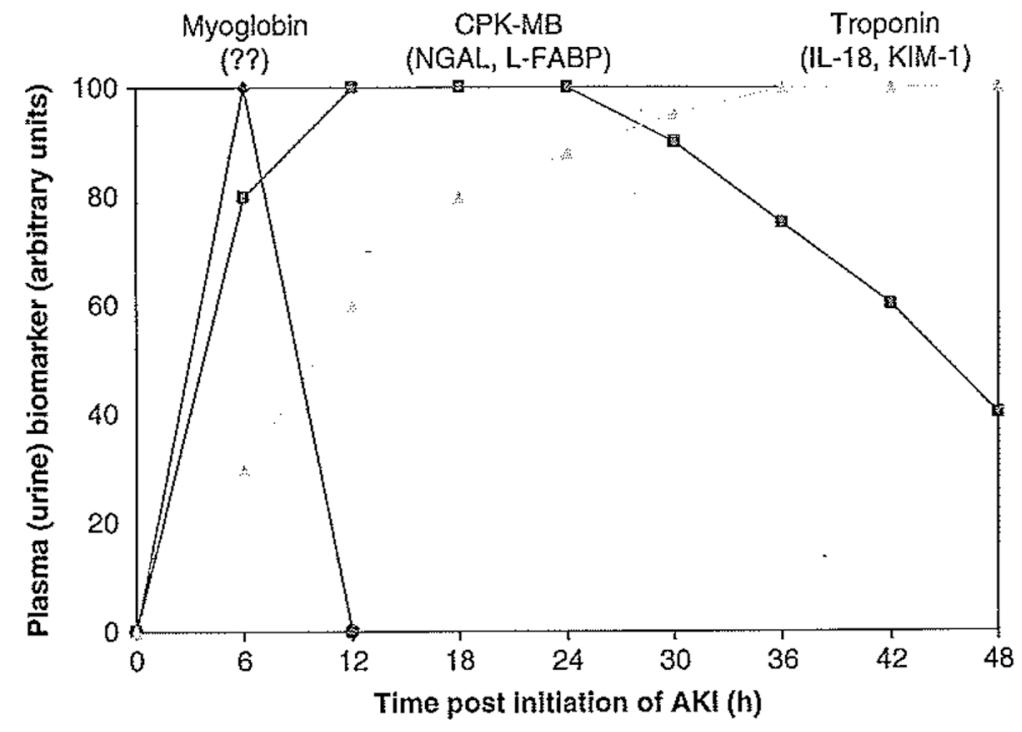

Figure 1. Proposed panel showing the temporal pattern of urinary biomarkers for the early diagnosis of AKI following defined events such as cardiac surgery and kidney transplantation, in analogy with cardiac markers in the plasma

AKI: Acute kidney injury; CPK-MB: Creatine phosphokinase; KIM-1: Kidney injury molecule-1; L-FABP; Liver-type fatty acid binding protein; NGAL: Neutrophil gelatinaseassociated lipocalin. 


\section{Table 1}

Current status of new urinary biomarkers for discriminating between different forms of AKI*.

\begin{tabular}{lllc}
\hline Biomarker name & CPB & Contrast nephropathy & Sepsis or ICU setting \\
\hline NGAL & 2 h post-CPB & $1-2$ days pre-AKI & 2 days pre-AKI \\
IL-18 & 6 h post-CPB & Not increased & 2 clays pre-AKI \\
KIM-1 & 12 h post-CPB & Not tested & Not tested hpost-tx \\
L-FABP & 4 h post-CPB & $1-2$ days pre-AKI & Not tested \\
& & & Not tested \\
\hline
\end{tabular}

The times indicated (in hours) are the earliest time points at which the biomarker is increased significantly from baseline values.

AKI: Acute kidney injury; CPB: Cardiopulmonary bypass; ICU: Intensive care unit; KIM-1: Kidney injury molecule-1; L-FABP: Liver-type fatty acid binding protein;

NGAL: Neutrophil gelatinase-associated lipocalin; tx: Transplant. 